\title{
Article
}

\section{An exploration of trainee practitioners' experiences when using observation}

Martin, E., Winter, S., and Holder, Tim

Available at http://clok.uclan.ac.uk/16815/

Martin, E., Winter, S., and Holder, Tim (2017) An exploration of trainee practitioners' experiences when using observation. The Sport Psychologist, 31 (2). pp. 160-172. ISSN 0888-4781

It is advisable to refer to the publisher's version if you intend to cite from the work. http://dx.doi.org/10.1123/tsp.2016-0019

For more information about UCLan's research in this area go to http://www.uclan.ac.uk/researchgroups/ and search for < name of research Group>.

For information about Research generally at UCLan please go to http://www.uclan.ac.uk/research/

All outputs in CLoK are protected by Intellectual Property Rights law, including Copyright law. Copyright, IPR and Moral Rights for the works on this site are retained by the individual authors and/or other copyright owners. Terms and conditions for use of this material are defined in the policies page.

\section{CLoK}

Central Lancashire online Knowledge www.clok.uclan.ac.uk

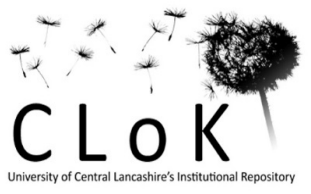


22 Date of re-submission: $13^{\text {th }}$ October, 2016 
Abstract

27 Observation provides applied sport psychologists with a direct assessment of client behavior

28

29

within the sporting environment. Despite the unique properties and the insightful information that observation allows, it has received limited literary attention within the applied sport psychology domain. The current study aimed to explore and further understand the observation practices of current trainee practitioners. All participants were enrolled on a training program towards becoming either a chartered psychologist (BPS) or an accredited sport and exercise scientist (BASES). In total, five focus groups were conducted and analyzed using an interpretative phenomenological approach (IPA; Smith, 1996). Four superordinate themes emerged: value of observation, type of observation, challenges of observation, and suggestions for observation training. Results demonstrate the increased value that observation brings to effective service delivery and intervention. Specifically, informal observation is commended for its propensity to build greater contextual intelligence and to develop stronger client relationships.

Keywords: applied sport psychology, focus groups, contextual intelligence 
An Exploration of Trainee Practitioners' Experiences When Using Observation Conducting a thorough and comprehensive needs analysis is an essential skill for an applied sport psychologist (Fifer, Henschen, Gould, \& Ravizza, 2008). Client information should be gathered both in the initial stages and throughout the consultancy period to ensure the appropriate identification of performance strengths and detriments, and changes in these (Tkachuk, Leslie-Toogood, \& Martin, 2003). Traditionally, interviews, questionnaires, and observations are triangulated to afford a holistic and increasingly accurate depiction of the performer (Hemmings \& Holder, 2009; Milne \& Reiser, 2011). As a result, relevant information is used to develop suitable interventions to facilitate constructive client change (Anderson, Miles, Mahoney, \& Robinson, 2002; Katz \& Hemmings, 2009).

Interviews and questionnaires are well represented in the applied sport psychology literature, and extensive guidelines are provided for their implementation (Taylor \& Schneider, 1992; Tkachuk et al., 2003). Two well established interview schedules include the sport clinical intake protocol (SCIP; Taylor \& Schneider, 1992) and the BASIC-ID framework (Davies \& West, 1991). The SCIP (Taylor \& Schneider, 1992) is intended to collate both sport specific and clinical information across seven areas considered to be important to the athlete's life. This includes: a) the presenting problem, b) life and athletic history, c) family and social support, d) health status, e) important life events, f) changes prior to the onset of the presenting problem, and g) details of the presenting problem.

Similarly, the BASIC-ID (Davies \& West, 1991) gathers both general and specific insight into athletic performance across seven modalities; behavior, affect, sensations, imagery, cognitions, interpersonal relations, and biological functioning (diet/drugs). In addition, current literature has advanced discussion to the principles of effective counselling skills, i.e., room set up, positioning, active listening, and relationship development (Katz \& Hemmings, 2009; Murphy \& Murphy, 2010; Sharp, Hodge \& Danish, 2015). 
In comparison, observation allows the applied sport psychologist to move beyond

typical one-to-one consultancies and become immersed into the rich, dynamic, and naturalistic settings of the sporting environment (Watson II \& Shannon, 2010). Every individual has a unique perception of their surroundings; hence it is imperative that sport psychologists observe specific behavioral issues within the client's specific sporting context (Orlick, 1989). Inevitably, observation is often criticized because of its inability to causally account for 'invisible' factors such as cognition and intention, and is therefore viewed as speculative (Gillham, 2008). However, it should also be recognized that standardized questionnaires present hypothetically fabricated scenarios, while interviews are generally conducted in an environment far removed from the client's sporting world. An explicit record of an individual's pattern of behavior (i.e. observation), is often dissimilar to an individual's perceived understanding of self-behavior and interaction (i.e. interviews; questionnaires) (Gillham, 2008). Considering this, it is essential for practitioners to be aware of the opportunities that effective observation can herald within the consultation process (Watson II \& Shannon, 2010).

Despite the ostensive need for observation there is limited research evidence into best practice, guidelines, and application in sport psychology (Baumeister, Vohs, \& Funder, 2007; Holder \& Winter, 2016). Typically, researchers have focussed on the implementation of formal observation via systematic instruments, aimed to count and record behavioral frequency in pre-determined categories (Gillham, 2008). To date there are two such instruments within the applied sport psychology literature, the self-talk and gestures rating scale (STAGRS; Van Raalte, Brewer, Rivera, \& Petitpas, 1994), and the multidimensional motivational climate observation system (MMCOS; Smith et al., 2015). While systematic observation instruments provide useful statistical information regarding the frequency of behavior, their limitations must also be acknowledged. The sporting environment is dynamic 
in nature and defined by contextual nuances that cannot be wholly represented by pre-defined and isolated behavioral categories (Hall, Gray, \& Sproule, 2016). Reliance on the simple quantification of behavior can result in a scenario devoid of context, limited in the richness of information gathered, and create missed opportunities to record behavioral triggers impacting performance (Cushion, Harvey, Muir, \& Nelson, 2012). Alternatively, informal observation considers the complexities of social interaction and requires the practitioner to become embedded within the sporting context (Gillham, 2008; Holder \& Winter, 2016). Nonetheless, despite both types of observation being used within applied service delivery; there is a stark indifference in the sport psychology literature regarding guidelines for its implementation when directly compared to traditional modalities of assessment.

The lack of literature and guidance pertaining to observation in applied sport psychology is surprising considering it is a cornerstone of assessment triangulation. This is particularly important when acknowledging that behavior plays an integral role within a wide range of philosophical approaches that steer applied sport psychologists in their service delivery, i.e., person-centered and humanistic perspectives, and cognitive-behavioural therapy (Fifer et al., 2008; Hemmings \& Holder, 2009; Poczwardowski, Sherman, \& Ravizza, 2004; Sharp et al., 2015). In this regard, it is of critical interest to gain a well-informed understanding of the client, which stems from a strong working relationship in which the applied sport psychologist is perceived as caring, genuine, and empathetic (Fifer et al., 2008). Observation of client behavior within the sporting environment is therefore a key avenue through which these attributes can be achieved. However, as a profession with accrediting bodies that champion evidence-based practice (Winter \& Collins, 2015a; 2016), applied sport psychology is surprisingly limited in both guidance and training of observation, and instead assumes that practitioners instinctively know how to observe (Holder \& Winter, 2016). 
Observation therefore appears to be a key area requiring professional development and understanding. Holder and Winter (2016) have begun to address these concerns through their exploration of experienced practitioners perception and use of observation. They specifically highlight the need for a greater evidence base, and as a result, we considered trainee practitioners as a relevant and important population to explore. The United Kingdom offers two training pathways towards attaining either chartered psychologist status and Health and Care Professions Council (HCPC) registration with the British Psychological Society (BPS), or accredited sport and exercise scientist with the British Association of Sport and Exercise Sciences (BASES). The nature of this training requires individuals to deliver, learn, and engage with psychological techniques under the supervision of an experienced practitioner. Consequently, this study aims to initiate an exploration into the observation practices of current trainee practitioners. Specifically, we seek to understand trainees' perceptions, justifications, and experiences of using observation.

\section{Method}

\section{Methodology}

Interpretative phenomenological analysis (IPA; Smith, 1996) is rooted within, and combines both phenomenology (descriptive element) and hermeneutics (interpretive element; Pringle, Drummond, McLafferty, \& Hendry, 2011). IPA reflects the authors' views of constructivism in which the individual and the world are viewed as co-constructing rather than two separate bodies, wherein the researcher plays an interpretive part (Davidsen, 2013; Palmer, DeVisser, \& Fadden, 2010). IPA was chosen as the qualitative approach for this study owing to the central position placed on an individual's lived experience and their resultant sense-making of these experiences (Smith \& Eatough, 2012). Alternative means of data collection have begun to adopt methods of IPA, namely focus groups (Palmer et al., 2010; Tomkins \& Eatough, 2010). Using an approach through which multiple perspectives 
of a given phenomenon can be shared is considered to uncover both implicit thoughts and subconscious opinion (Tomkins \& Eatough, 2010). As a result, focus groups can build a deeper experiential understanding of a given phenomenon due to the dynamic interplay between participants (Liamputtong, 2011).

\section{Participants}

Supervisors associated with either the BPS or BASES, were identified via their accrediting body's website, and emailed to request permission to contact their supervisees. Primary contact with supervisors was intended for recruitment of individuals from the same supervisory group. It was considered that participants sharing the same supervisor were likely to have had previous group reflections and would not therefore be averse to sharing knowledge and experiences in a discursive environment. In some instances supervisors did not run group supervision; however this method of contact enabled greater ease when organising focus groups due to participants residing in relative proximity to each other. In total 16 supervisors across England and Wales were initially contacted. Due to non-response, or difficulties organizing a suitable date and time for all individuals, the final participant sample represented eight supervisory groups. All participants were enrolled on a training program towards either chartered psychologist (BPS) or an accredited sport and exercise scientist (BASES). The final sample of participants represented a homogenous group to align with IPA guidelines (Smith \& Eatough, 2012). Across the sample were minor discrepancies between participants, including group and individual supervision, as well as variation in the stage of training, i.e., at the start, middle, and end of this process. Following institutional ethical approval and informed consent, the sample included seven males (age: M $=31.71$ years, $S D=9.32$ years), and 12 females (age: $\mathrm{M}=25.33$ years, $S D=1.37$ years), creating five focus groups, comprising between three to five participants. Liamputtong 
(2011) and Litosseliti (2003) advise that smaller participant numbers in focus groups elicit greater contribution from each member, allowing for better articulation of opinion.

\section{Focus Group Design}

A natural human behavior is to discuss perceptions and opinions of specific topics in a group setting; consequently focus groups appeal to the ordinary conversation and social interaction of everyday life (Colucci, 2007; Litosseliti, 2003). Heightened interaction and sense making between participants therefore create opportunity to uncover implicit perceptions regarding observation experiences (Liamputtong, 2011; Tomkins \& Eatough, 2010). To align with the principles of IPA, the researcher adopted the role of a moderator and ensured that focus groups were participant-led (Smith \& Eatough, 2012). Through assuming a position as moderator, the researcher can actively engage participants and encourage discussion between group members, rather than between moderator and participant (Liamputtong, 2011).

A pilot focus group was conducted by the first author and observed by the second author, to ensure wording and ordering of the question guide and stimulus activities were clear, unambiguous, and logical. Consequently, stimulus activities and ordering of questions were perceived to be effective in evoking a suitable and relevant depth of discussion.

However, the first author's style of questioning was considered too directive, and was therefore adapted to accommodate an approach more suited as a facilitator of group discussion. This ensured the power relationship held between researcher and participants was in favour of the participants, giving them a voice when exploring phenomena, and which supports an IPA approach (Hopkins, 2007).

Each focus group followed a semi-structured question guide with stimulus activities integrated throughout (available upon request from the first author). The premise of stimulus activities is to provide an alternative technique in which to provoke discussion and elicit 
answers while increasing the comfort, relaxation, and enjoyment of participants (Colucci, 2007; Liamputtong, 2011). In effect, activities are aimed to maintain participant attention, and to promote the flexibility of participants to freely discuss and explore their observation practice and experiences. Three stimulus activities were included. Firstly, an ice-breaker encouraged all participants, inclusive of reserved characters, to contribute from the outset to group discussion (Liamputtong, 2011). Secondly, a free-listing task promoted participant autonomy and provided an opportunity for group members to discuss personally perceived areas of interest rather than being constrained by a listed itinerary. This facilitated the recognition of shared or common experiences between participants, which strengthens developed ideas or concepts (Palmer et al., 2010). The final stimulus activity was a problemsolving task designed to encourage innovation.

The question guide included an introductory question, i.e., "What are your current experiences of using observation when assessing a client?", transition questions, i.e., "How do you know how to observe?", and a focus question, i.e., "What are your perceptions of observation?" The last two stimulus activities were implemented at this stage of the focus group to generate further discussion. Following this was a summarising question, i.e., "If you were given the opportunity to have formal training in the application of observation, what would you like included?", and a concluding question, i.e., "Is there anything else that you wish to discuss?" (Liamputtong, 2011). Additional probes were used to encourage participants the opportunity to expand upon responses.

\section{Procedure}

Participants were emailed an information sheet by the lead researcher, regarding the purpose of the study. All were made aware of their rights to abstain from the study at any point, and were asked that all information shared within the focus group be held confidential (Willis, Green, Daly, Williamson, \& Bandyopadhyay, 2009). Following informed consent, 
participants were emailed as a collective to decide on the most convenient time and location for their respective focus group. Each focus group lasted approximately 75 minutes $(M=$ $76.25 \mathrm{~min}, S D=14.08 \mathrm{~min})$.

\section{Data Analysis}

Analysis of data was based on IPA guidelines published by Palmer et al. (2010). Focus groups were videoed, audiotaped, and transcribed verbatim using pseudonyms to protect the identity of participants. The use of video is recommended in the transcription process to identify the speaker, as often an audiotape can be unclear if multiple individuals are speaking simultaneously (Hopkins, 2007). Raw data was comprised of 203 pages and 6,543 lines of verbatim transcript. Transcripts were line numbered to help with the location of specific contributions at a later date. In the left hand margin notes were made regarding reflective questioning of the contribution. Reflective questioning and resultant interpretation of data referred to notions such as participant positionality, which specifies the participant's relationship with the matter of concern, therefore contextualising the data. In the right hand margin contributions were labelled and later organized into emergent patterns. Data was analyzed by the lead researcher on two separate accounts, firstly at a group level, with resultant superordinate and subordinate themes, and secondly re-analyzed and interpreted from each individual's perspective. This ensured that data was not occluded at either the group or participant level and provided a holistic interpretation of deeper experiential accounts (Palmer et al., 2010). An IPA approach stipulates that results are strongly associated with the dialogue of participants (Pringle et al., 2011). Quotes were thus selected based on how successfully they represented the group discussion and resultant emergent themes.

\section{Establishing Quality and Rigour}


IPA recognizes the researcher as integral to the collection of data and its resultant interpretation; therefore the subjectivity of the researcher's own experiences and understanding of the world has the potential to affect the research process (Pringle et al., 2011; Sparkes \& Smith, 2014). To help identify any implicit bias and to focus the researcher's understanding of their own impact on the developing content, a reflexive journal was kept (Etherington, 2004). This acted as a learning tool for the lead researcher and enabled the double hermeneutic (i.e., making sense of the researchers own interpretation, whilst also making sense of the participants interpretation) of an IPA approach to be addressed (Vicary, Young, \& Hicks, 2016). Transparency was integral to ensuring the rigour and quality of data. To achieve transparency the lead researcher kept a clear audit trail of steps taken towards data collection, annotation of transcripts, and its resultant interpretation which was shared and discussed with the secondary researcher (Palmer et al., 2010; Vicary et al., 2016). Keeping a reflexive journal was also considered as a form of bracketing, due to its nature of reflective practice and resultant questioning of both the participants, and researchers positionality relative to the topic in discussion (Palmer et al., 2010; Vicary et al., 2016). Together, these processes ensured constant monitoring and mindfulness of the researcher's unintentional impact upon the research process (Patton, 2002).

\section{Results}

Trainee practitioners' experiences of observation in applied practice elicited numerous subordinate themes resulting in four superordinate themes: value of observation, type of observation, challenges of observation, and suggestions for observation training.

\section{Value of Observation}

Observation was identified to positively enhance client understanding and knowledge of the sporting environment. As a result four subordinate themes emerged: triangulation, confirmatory evidence, contextual intelligence, and development of relationships. 
Triangulation. The traditional approach of triangulating data across all assessment types (i.e., interviews, questionnaires, observations) was stated as a valuable technique towards developing suitable and effective client intervention. However, an alternative means of triangulation was also identified:

Kate: ...so ask the coach how they think the player is and what their behaviors are....are there any issues or anything like that...Ask the client; obviously you've then got your observation and if you can ask the parents as well because I can guarantee all three or all four are going to be different in some way.

Faye: Yea...because if you go and watch one session you don't know if that's a typical session...or whether actually that's a really abnormal session...so asking other people helps like you say to triangulate. (FG3)

Faye recognizes that sole reliance on one representation of the client may not characterize typical client behavior. Comparison of judgements from significant others was perceived as important in generating a fair depiction of behavioral tendencies.

Confirmatory evidence. Observation can verify the extent to which the client's perceived account compares to the explicit record of behavior demonstrated:

Faye: And do you think it gave you more information because the anger thing had come up in one to one conversations but... actually you hadn't maybe realized how bad it was? Kate: Yea you can see it clearly. So they talk about it and you think ok well how are they getting it into perspective? Because it never reached that level in our conversations. So to actually see it at one of the highest intensities... where he was physically going to start a fight...

George: And I guess it's allowed you to pinpoint a little bit about what the triggers are more specifically by observing that situation. (FG3) 
Sense making between participants demonstrates that observation is perceived as an exclusive means of attaining first-hand evidence otherwise inaccessible if reliant on only primary assessments (i.e., interview). Terms such as, 'hadn't...realized', 'getting it into perspective', and 'it never reached that level in our conversations' suggests the intensity of emotion can only be witnessed through observing in the sporting environment. Further, observation has the capacity to inform and alter an existing perception assigned to a client: ...my opinions and perceptions I guess were flipped in a lot of cases...I couldn't believe...all the inaccurate judgements and perceptions that I had made, because yea ok that's how they are in a classroom but that isn't where they play football. (Ashleigh, FG1) Interpretation suggests that it was only through observation that Ashleigh was able to become more critically aware of initial judgements to confirm or disconfirm original client perceptions. Without observing there is significant opportunity to be misguided.

Contextual intelligence. Presence in the sporting environment was considered to be of invaluable reward:

Faye: I think that's a really good point, learning about the sport...So actually yes you're there to observe the athlete, but you're also there to get to know more about the sport. Especially if it's one that you don't know much about already...

Kate: It's about culture isn't it?

George: Yea I don't think I learnt anything about the athlete per se really apart from it looked quite good because I didn't really know what I was looking for...you learnt about the setup of it, where they go, when they get there, what the behaviors are...maladaptive behaviors are, as well from the crowd...I probably learnt quite a lot here. (FG3) Continual reference to the word 'learning' indicates that observation creates a unique opportunity to gather information and create a greater implicit understanding of the mechanisms and intricacies of the sporting culture. It can be interpreted that observation in 
this sense is informal, and subliminally builds cultural knowledge to overcome many challenges associated with working in a new sport, or with a new client.

Development of relationships. Observation is largely reported to help build and develop relationships with the client, coaches, and team:

Lee: ... it was about minus four and I was stood there on the side-line and I didn't really have anything in particular that I was going there with a view to observing...it was more just being around and if they needed to speak to me or anything...about four or five girls came over and said God you must be bloody freezing, you know are you alright? But that showed me obviously they had noticed that I'd bothered, just to show them that I cared was a big factor as well. That made me feel pretty good that they'd noticed it...So that carries a lot of weight as well when you're working with people.

Aoife: Yea I've had that as well...that kind of shock from an athlete...you want to come...are you looking for anything? No I just want to see... what you go through within your training sessions...your interactions with your coach, different things like that, but massively thinking that oh she cares what I'm doing. (FG 5)

Lee and Aoife demonstrate the significant impact that 'just being around' has on the development of client relationships. Both described how their presence as trainees in the coaching/performance environment came as a shock to their clients, suggesting that such behavior was unexpected and outside of the more evident role of traditional consultancy. Interpretation suggests that a practitioner's explicit display of will, enthusiasm, and interest was linked to gaining client rapport and respect, facilitating integration and acceptance into the sporting environment. 
Two subordinate themes emerged from the data: formal observation and informal observation. It is suggested both types of observation serve distinctly different purposes for the trainee practitioner when gathering information.

Formal observation. Information gathered from previous client assessments (i.e., interviews) was perceived to better structure observation due to the pre-identification of target behaviors: “...you're going in because you've spoken about something and you're looking to observe certain behaviors" (Aoife, FG5). When targeting specific behaviors, an observation instrument was deemed desirable: determined behaviors were perceived by some, as detrimental to effective observation: 
to do with it all, like what the point was at the end. I...sort of just abandoned it." (Anita, FG5). From this is can be inferred that keeping a frequency count of behavior becomes meaningless data which does not detail enough of the client's story. Supporting this perception, it was recognized that an observation instrument within a team setting of multiple variables is largely more complex than using it with an individual client:

Lee: I think it would be a hell of a task if it was a different sport, more fluid situation... you just wouldn't be able to do it.

Anita: Would you be able to do it even in a team situation, because that's a lot of...there's so much that you need to do it sounds like.

Lee: You'd struggle yea.

Hannah: You'd need about ten different people!

This excerpt suggests the success of using an observation instrument to formally observe is largely dependent on the situation and context in which it is used. The tone and humour of this last claim insinuates the group's belief that using a systematic observation instrument would be cognitively arduous when observing multiple individuals simultaneously.

Informal observation. This type of observation is characterised by being there, hanging around, being in the background, and face time (to be visibly seen), and forms an increased presence in the client's sporting environment: ...you're there and you're observing but you forget that you are observing but you are because you're consciously thinking about things, you're deliberately watching certain players, certain behavior, certain interactions, yet you forget that you're doing a form of psychological assessment... (Ashleigh, FG1) Ashleigh recognizes that observation can be forgotten as an assessment, despite it being a deliberate and conscious process. Alternatively, it could be interpreted that for Ashleigh, informal observation is becoming implicit and continual in her role as a trainee practitioner, 
and therefore she is not always aware of the valuable information it can elicit. The following conversational excerpt supports the notion that informal observation can be forgotten as a valued assessment:

Janine: ... you have to find that purpose as to why you're sat there and watching. Is it for observations or are you just sitting there and just because you're in the environment you're just watching it. So it's the difference between observations...

Charlotte: and just watching.

Janine: ...and actually structured or just...

Natalie: ...just there. (FG4)

Interestingly, sense-making between participants uncovers the concept of there being a difference between 'just' watching and observing. The use of 'just' as a prefix to watching suggests it is not a skilled process, as opposed to observation which is considered a structured and skilled practice. This advocates that, if used effectively, informal observation is a purposeful and valued opportunity to collate information through an increased presence in the client's sporting environment:

Aoife: ...that's an example of how just being there as well...you're not directly observing them having a conversation, but by being there and overhearing something then you have a bit more insight into the context for when you do have to sit down and have a one-toone with someone.

Lee: And you get some random things that pop up out of nowhere that you're not expecting...you're just stood there and someone will come over and just mention something off the cuff. (FG5)

As a result it is inferred that informal observation facilitates the value of observation via building both contextual intelligence and developing relationships.

\section{Challenges of Observation}


Engagement in observation presented associated challenges. As a result four subordinate themes emerged: lack of observation guidelines, trainee preconceptions and perceptions of others, logistics of observation, and distraction during observation.

Lack of observation guidelines. The majority of participants stated to have received no formal observation training:

Natalie: I think everyone just assumes that because it's called observation you just watch... and they think it's self-explanatory but it's probably not.

Janine: Yea. No in terms of skills wise I've never had anything...

Charlotte: No I don't think we have.

Janine: ....any guidance at all.

Charlotte: I think you probably, like you said, you think it's just simple, you're just watching someone. (FG4)

As conversation unfolded, participants began to re-evaluate initial assumptions of observation and speculated these as inaccurate, indicating an area warranting further consideration. As a profession advocating that applied work be underpinned by an evidencebase, it is expected there should be sufficient reading material regarding observation practice: ...we're doing evidence-based practice and that your practice should be underpinned by what research is saying. So then for me I was like ok, surely there's some applied practice research around the use of observation as a sport psychologist and I was quite shocked that there wasn't as much as I wanted there to be. (Ashleigh, FG1)

The surprise elicited at discovering insufficient observation guidance in the literature could be interpreted that as a valued assessment there is an expectation that observation practice should be grounded in an evidence-base. Without it our understanding and resultant effectiveness is limited: 

...for us that have been on it [supervised experience], for a couple of years to somebody that now's coming into it. We're probably pretty much at the same level in terms of...real structure and knowledge of observation. I can pretty much safely say that I've not had any, even with my supervisor; we hadn't really discussed observations in any way at all. (Janine, FG4)

It is considered perturbing that early career professional development is severely hindered without the opportunity to access training or guidelines. Janine may not have made this association without the sense-making and interactive interplay of participants sharing lived experiences.

Trainee preconceptions and perceptions of others. Trainees noted caution regarding potential preconceptions when interpreting client behaviors:

Ashleigh: Do you think that interferes with your observations from an applied sport psychologist's point of view because sometimes I feel that I have that in my advantage with the sports I'm working in because I haven't coached in them. But then I think if I was doing an observation of a track or field athlete which is my sport, which I'm a qualified coach in, then I probably would have a bit more of a coaching head on... Alex: I think I would be able to draw the line and say right, no I shouldn't be thinking about that, that's tactics, that's technical stuff, focus on something else. However, I think that there will be a fuzzy line in between the two at some point that would say right, which skill set am I tapping into. (FG1)

This excerpt highlights trainees challenging and critically questioning each other's potential preconceptions. It is essential to engage in such practice to ensure preconceptions do not influence the interpretation of client behavior.

Analysis of data reveals a pre-occupation of trainees to project a positive impression of themselves to the client: 
Janine: ...coaches observe that all the time so it's not like it's a process that players aren't used to, it's almost a connotation with psychologists that can cause more barriers with the observations for us than it necessarily would with a coach...

Charlotte: I think that might actually be one of the reasons that I don't use it as much, is that inability to explain it to the player's, because a lot of my research has been on people's perceptions in sport psychology so I know how easily affected people's perceptions can be. If they've had one bad experience then that's it, they never think sport psychology can help them. So I'm very conscious to give people a good impression of sport psychology. (FG4)

It can therefore be suggested that trainees' fear of negative client perceptions stem from their own hesitancy and lack of ability to appreciate and advice on the value of assessments such as observation.

Logistics of observation. Observing in circumstances where there are multiple variables occurring simultaneously, typically a team environment, proves difficult, as Eric (FG2) states: "...just that physical process of capturing the data...could be overwhelming." The realism of observing in such an environment is that it is not always possible to watch the entire team training in one location:

Matthew: ...you might have a team in another team. You have the front row doing a completely different job to the second row...and then you might find that backs go somewhere else and forwards go somewhere else and suddenly...

Louise: You can't be in two places at once.

Matthew: You've got to decide. It's about decision making as well. (FG2) Both examples magnify the complexity of observing a team. As a result it could be inferred that observation is a skilled practice that requires professional judgement and effective decision making. Similarly, positioning oneself effectively within a sporting 
environment was often cited as problematic due to acoustic and visual challenges creating potential for missed information. Some sports were considered more challenging than others (i.e., road cycling), in which it was suggested that observation take place pre or post performance. Likewise, access to the competitive environment may alter the typical layout and rules of engagement the practitioner is familiar with, i.e., sitting with the spectators some distance away from performers, or not having access to a team during half time.

A final logistical challenge to emerge from the data was the prospect of charging for observation:

...even though it's part of my work I don't know how I would feel comfortable to say right I'm coming to observe you for this training session and I'm going to charge you an hour's rate or whatever. I just think I would feel really uncomfortable but it is part of your job and if you value it then should you feel uncomfortable? (Ashleigh, FG1) Prospective charging is met with caution and anxiety despite advocating its centrality to the job.

However, trainees associated working for an organization as an enabler for frequent observation, as evidenced by Ashleigh (FG1): “...it facilitates and encourages...the use of observation...a lot more just because of the nature of the organization of the much more scheduled sessions." It was suggested across focus groups that prospective charging for observation becomes easier when employed by an organization due to the capacity to block consultancy work into $x$ amount of hours as opposed to charging by the hour. Charging for blocks of time was perceived to empower the practitioner to use this time as they see appropriate.

Distraction during observation. Distraction emerged as a challenge towards staying focused for the entirety of an observation: 
George: ...because I like sport so much, particularly certain sports...becoming preoccupied by events of what actually happens during a game...you just think...oh the passing in this game is really good or, their skill levels really good. You find that you've been watching for minutes and you're not really looking for what you're looking for... Faye: ...no I find that for hockey and the rapport is good, but I think almost the more rapport you get...with someone or a team in my case...you really want them to win... and then you get really caught up in the game and, like you say then you're not actually doing your job and observing. (FG3)

Enjoyment of the sport has the potential to lead to spectatorship as opposed to observation as a practitioner, which is suggested to become particularly difficult when a relationship has been developed with the client. This excerpt implies the difficulty in maintaining professional boundaries when developing a rapport with clients, and being aware that presence in the sporting environment requires focused attention.

Alternatively, the realism of observing is such that being attentive over long periods of time is cognitively strenuous:

Eric: ...you might go somewhere and watch something and it's just, you find it's not interesting. It's boring...Committing to do the job properly as much as anything else... Ben: It's taxing on the mind. It definitely is.

Louise: Especially if not a sport that you're completely and utterly interested in. (FG2) Reference to being 'committed to do the job properly' implies the demanding nature of observation, and the need to approach it in a dedicated and proficient manner.

\section{Suggestions for Observation Training}

Trainees were receptive to proposed formal training in observation, resulting in two subordinate themes: shared experiences of observation, and proposed content for observation training. 
Shared experiences of observation. Consensus across focus groups stated that

experiences of observation from individuals currently on an accreditation training pathway, be shared with skilled practitioners with a number of years' experience: Anita: ...people who have just started out they sometimes have a different perspective on those that have been doing it for years. So you can see the differences as well and it's sometimes a bit more reassuring to see what people at your level are doing.

Aoife: That's why I think workshops are good because you'll get a range of people doing it and if the practitioners that run them are working in the field and are engaged in observation you get their input but then also as a group you can share experiences. (FG5) Numerous suggestions were posited regarding the range of experienced practitioners; including those that have worked in a number of sports, both individual and team, performance analysts, coaches, and coach educators. Alternatively an insightful suggestion was to include practitioners’ from a range of philosophical backgrounds: “...if you were from a humanistic point of view you might have a different observation than someone from CBT or positive psychology..." (Matthew, FG2)

Open enthusiasm to hear from a range of individuals with differing levels of experience and philosophical background indicates the receptive nature of the group of participants to share experiences, and create a co-constructed learning environment.

Proposed content for observation training. The primary suggestion of training content was unanimous across all focus groups and is reflected below:

Lee: ...you'll hear people talk constantly about the benefits of this or theoretically why that's underpinned with this but they don't actually talk about the tangible evidence or examples of why...Or how, this is what I actually do.

Hannah: That's the hard bit isn't it? 
Lee: ...that's the bit we need isn't it? Because we're loaded up with theoretical...but we want to know in the real world what you actually do... and that's where the anxiety is for me. It's the fear of the unknown.

Anita: Especially when you're just starting out as well because...you have no experience... (FG5)

It was suggested that proposed training be directed towards how observation can be applied in practice rather than an emphasis on theoretical underpinning. The tone of this conversation can be interpreted as frustration caused by limited availability of practical recommendation to aid effectiveness of service delivery.

Overall, the results depict an interesting overview of the trainee population and their understanding and use of observation practices. As indicated throughout, there appears to be overlap between subordinate themes, namely the facilitative effects that informal observation has on enhancing contextual intelligence and the development of relationships. The sensemaking of group members has been portrayed through the representation of conversations where most appropriate. From this, the reader can gather an understanding of group members' supportive claims, implicit opinions, and critical challenges of each super and subordinate theme.

\section{Discussion}

The current study aimed to explore and ultimately contribute towards an increased understanding of the observation experiences of trainee practitioners. Findings have provided an insightful perspective of individuals' observation practices within their early career. It must be noted however, that experiential claims and resultant themes are relative to the trainee population, and therefore those practitioners with greater experience are likely to face different challenges. It is also important to recognize that although results are segmented into separate superordinate themes, when representative of applied practice these are interwoven. 
Specifically, the underpinning superordinate theme, type of observation (i.e., formal or informal), has an associative impact upon the themes, value of observation, and challenges of observation. It is encouraging that current findings support existing understanding that formal observation plays an essential role within triangulation and confirmatory evidence (Milne \& Reiser, 2011). However, perhaps the most significant finding to have emerged is the perceived beneficial role that informal observation has for effective service delivery, characterized by 'hanging around', 'just being there', and 'face time'.

Traditionally practitioners spend significant time building extensive application-based knowledge regarding psychological skills and techniques (Brown, Gould, \& Foster, 2005; Hays \& Brown, 2004). Although it is important to be sufficiently knowledgeable in this way, it only provides a myopic understanding of the client, which poorly reflects the reality of the performance environment (Kutz \& Bamford-Wade, 2013). The environment in which an individual operates (i.e., the client), offers a landscape that is continually shifting and volatile due to the dynamics and interactions of agents (i.e., performers, coaches, values, culture) constituting a socially multifaceted setting (Kutz \& Bamford-Wade, 2013). Considering the unpredictable nature of the sporting environment it is imperative to build operational knowledge (i.e., contextual intelligence) via immersing oneself into the environment (i.e., informal observation), which facilitates an intricate understanding of the context and resultant culture in which the client operates (Brown et al., 2005; Winter \& Collins, 2015b).

Trainee concerns of gaining entry within an organization, lack of sport specific knowledge, and apprehension of stigma attached to sport psychology could be successfully addressed through building contextual intelligence. Immersion into a team's environment via increased informal observation enables the trainee practitioner to identify sporting rules while gaining familiarization of its language (Holder \& Winter, 2016). Hays and Brown (2004) compare learning a sporting language to entering a foreign country for the first time. In this 
context it does not matter how intelligent one is, if they do not possess an awareness of the contexts custom, culture, local language, and history there will be an issue with transference of said intelligence and its effectiveness. Hence, the combination of contextual intelligence and immersion create a powerful formula towards a practitioner becoming co-lingual and responding to the client's reality in their cultural context to ensure successful intervention (Brown et al., 2005; Kutz \& Bamford-Wade, 2013). Only through spending considerable time informally observing in the clients environment across multiple settings are practitioners afforded the opportunity to develop such intelligence.

As a consequence of contextual intelligence and immersion, the practitioner is in an optimal position to target and interact with relevant individuals (i.e., client, coach, manager) to develop and strengthen relationships. Practitioner immersion via shadowing the coach, attending training camps, and travelling to competitions both home and away are deemed to gain greater respect and trust from both the client and organization hierarchy (Brown et al., 2005; Fifer et al., 2008; Partington \& Orlick, 1987). As such, developing relationships is deemed inherently influential in attaining a collaborative and effective working partnership (Lubker, Videk, Geer, \& Watson, 2008).

First impressions of a practitioner are considered crucial in influencing an individual's perception of the sport psychologist's professional capabilities and resultant motivation to collaborate and seek help (Lubker et al., 2008). Given this, it is not surprising that client and coach's perception of the sport psychologist emerged as a consistent concern for trainees. Fear of being perceived negatively caused anxiety and adaptive behavior from trainees during formal documentation of observation. As one trainee described they did not want to be perceived as 'the weirdo in the background' while observing. Others also state they changed their behaviors in an attempt to conceal they were observing, by hiding note taking, or 
standing in a position where they are less noticeable. Causes for such behavior were stated to avoid negative client perception of being watched, and its resultant impact on client behavior. However, the question that must be considered is whether the extent of this associated anxiety is a result of an outdated belief. Historically, the perception of sport psychologists' has been blanketed with a stigma declaring practitioners' as either 'shrinks' due to a misconception that sport psychology is deemed the same as psychotherapy, or as 'ivory tower' consultants whose concern is with science as opposed to applied work (Barker \& Winter, 2014; Partington \& Orlick, 1987). Encouragingly, there appears to be a shift within the literature that acknowledges an emerging positive perception of sport psychology from athletes (Pain \& Harwood, 2004). Observation particularly has been stated as a highly valued experience that develops empowerment and satisfaction for both the client and practitioner (Madan, Conn, Dubo, Voore, \& Wiesenfeld, 2012). Despite this, there are still negative attachments to the term sport psychology creating barriers for practitioners' capacity to fully integrate within a team (Pain \& Harwood, 2004). Often this negative perception does not originate from the client, but rather from a concern of what others (i.e., teammates) would think if they were to seek help (Blom, Hardy, Burke, \& Joyner, 2003). It is believed that negative perceptions are a result of poor education about the services offered by applied sport psychologists', therefore it is encouraged to ensure clients are appropriately educated in the positives of consultation, i.e., observation (Pain \& Harwood, 2004). Alternatively, 'hanging around' via informal observation in the early stages of a collaboration may informally educate coaches and performers in understanding the role of the sport psychologist and challenge any negative stigma attached to the profession.

Although informal observation has the potential to be influential in alleviating some challenges of observation (i.e., perceptions), it is important to recognize that other challenges are inherent within observation and are difficult to overcome. Namely these are logistical 
barriers such as, positioning, environmental access, financial and time costs. Predominantly it must be stressed that observation is acutely demanding on time, particularly if practitioners have other working roles, as trainees have alluded to (Madan et al., 2012). In such scenarios, although informal observation can be deemed defunct due to its costly nature in time, it must be argued that such an assessment should be encouraged where possible due to the valuable information and opportunities that 'hanging around' can provide the practitioner and their resultant service delivery. Alternatively, when time becomes a challenge practitioners may benefit from using formal observation. It can be argued that formal observation is a more efficient use of time due to being a direct assessment that provides tangible evidence of client behavior.

Observation is regarded as a technique that requires training to develop skill that allows the practitioner to monitor, acknowledge, and respond to behavioral changes within a continually shifting environment (McMorris, 2015). Interestingly, focus group four distinguished between watching and observing. Observation was labelled as a pro-active, structured and purposeful activity. Current findings imply that formal observation is purported to gather tangible evidence and documentation, while informal observation is used to attain contextual intelligence via immersion into a sporting culture, leading to relationship development. Watching however is considered a reactive activity and is therefore not skilled. Insightfully Matthew (FG2) claims practitioners should be: “...view[ing] something that's not just seen by other people" suggesting observation is a skilled practice that should be learnt and deliberately applied.

Considering observation is a skilled practice it is alarming to find trainees have received minimal to no formal training in it. Evidence from trainees suggests they are implementing observation; however there is resounding indication this is founded on instinct and trial and error learning, rather than evidence-based practice as is endorsed by governing 
691

692

693

694

695

696

697

698

699

700

701

702

703

704

705

706

707

708

709

710

711

712

713

714

715

accreditation bodies (i.e., BPS and BASES). It is fair to assume that at this stage of a trainees' career, observation skill is relatively low, therefore training in this much valued assessment should be essential. Proposed formal observation training was met with unanimous consensus across all trainee participants, explicitly showcasing the merit in providing such a platform of education. Most salient was the suggestion from trainees to focus on the how of observation as opposed to what and why. This conviction is supported through Brown et al. (2005) claiming current applied sport psychology training as being proficient in the development of individual techniques (i.e., what and why), but which is not adept at educating practitioners in navigating the complexities of an ever-changing sporting context (i.e., how). It is anticipated the sharing of experiences and knowledge from a varied range of practitioners, will enable reflective discussion and take home messages regarding associated challenges such as, coping with the demand of multiple variables (i.e., teams), distraction, documentation, and perception. Attention should also be given to developing an understanding of contextual intelligence and its propensity to open doors to generate greater effectiveness in applied delivery.

Practical recommendations for enhancing observation practice have been interspersed throughout this discussion. We feel it is critical that our clients are educated on the purpose and intention of observation in an attempt to dispel any negative association or discomfort linked to being observed. By selling its positive implications, it is anticipated that applied practitioners will be less inclined to conceal observation, and instead confidently observe and integrate themselves into the sporting environment. Resultant immersion into the client's environment is recommended to help build a contextually intelligent practitioner. An increased presence, via informal observation, facilitates a deeper understanding of the complexities of a specific sporting culture, allowing practitioners to more effectively design and implement intervention. Furthermore, it is recommended to be seen in the sporting 
environment outside of 'normal' working hours in an outward display of enthusiasm and interest. Both the client and key stakeholders within the sporting organization are likely to be recognisant of this which is suggested to increase acceptance and strengthen relationships.

It is important to reflect on possible limitations of the study. Consideration is given to the combined participant sample of individuals supervised from two different accreditation systems with differing outcomes and training structures (i.e., BPS and BASES), and the potential impact this may have had on individual experiences. Reflecting on each focus group and their responses to questions regarding previous observation training, it was considered that all participants, regardless of background or training pathway produced similar answers. Thus authors felt that all participants had similar foundations in observation knowledge irrespective of which accreditation system they were affiliated with, and therefore the differences in supervision across both training pathways would be minimal. Secondly, it is recognized that a wider group of trainees from a bigger sample of supervisors across the United Kingdom may provide a better representation than drawing from a small cluster of supervisors. Lastly, it is important to consider the impact of researcher bias on the interpretation of data. Due to the relatively small community of trainee practitioners' within the United Kingdom, the lead researcher had professional connections with some of the participants used in focus groups. However, to minimize the effects of potential researcher bias, a reflexive journal was kept in which the lead researcher acknowledged any bias and unintentional influence upon data collection and interpretation.

\section{Conclusion}

The most influential message to have emerged from this study is that observation is perceived to add substantial value to service delivery, which is currently vastly under acknowledged. Positive properties already associated with observation, such as triangulation and confirmatory evidence (Watson II \& Shannon, 2010) has been largely reinforced, 
741 however findings have uncovered other significantly valued traits of observation. A running

742 undercurrent throughout this discussion has been the role of contextual intelligence as an

743 avenue worthy of much greater exploration due its potential for unlocking many of the

744 perceived challenges already attached to observation. Exposure and raised awareness of

745 these associated challenges (i.e., trainee preconceptions and perceptions of others) is

746 imperative for the advancement of our profession. Future research should be directed

747 towards the development of observation training if the profession of applied sport psychology

748 is to aspire and develop towards an ever effective and successful discipline.

749

750

751

752

753

754

755

756

757

758

759

760

761

762

763

764 
767

Anderson, A. G., Miles, A., Mahoney, C., \& Robinson, P. (2002). Evaluating the effectiveness of applied sport psychology practice: Making the case for a case study approach. The Sport Psychologist, 16, 432-453.

Barker, S., \& Winter, S. (2014). The practice of sport psychology: A youth coaches' perspective. International Journal of Sports Science and Coaching, 9, 379-392.

Baumeister, R. F., Vohs, K. D., \& Funder, D. C. (2007). Psychology as the science of selfreports and finger movements: Whatever happened to actual behaviour? Perspectives on Psychological Behaviour, 2, 396-403. doi:10.1111/j.1745-6916.2007.00051.x

Blom, L. C., Hardy, C. J., Burke, K. L., \& Joyner, B. A. (2003). High school perceptions of sport psychology and preference for services. International Sports Journal, 7, 18-24.

Brown, C. H., Gould, D., \& Foster, S. (2005). A framework for developing contextual intelligence (CI). The Sport Psychologist, 19, 51-62.

Colucci, E. (2007). "Focus groups can be fun": The use of activity-oriented questions in focus group discussions. Qualitative Health Research, 17, 1422-1433. doi:10.1177/1049732307308129

Cushion, C., Harvey, S., Muir, B., \& Nelson, L. (2012). Developing the coach analysis and intervention system (CAIS): Establishing validity and reliability of a computerised systematic observation instrument. Journal of Sports Sciences, 30, 203-218. doi:10.1080/02640414.2011.635310

Davidsen, A. S. (2013). Phenomenological approaches in psychology and health sciences. Qualitative Research in Psychology, 10, 318-339. doi:10.1080/14780887.2011.608466

Davies, S., \& West, J. D. (1991). A theoretical paradigm for performance enhancement: The multimodal approach. The Sport Psychologist, 5, 167-174. 
TRAINEE EXPERIENCES OF OBSERVATION

791

792

793

794

795

796

797

798

799

800

801

802

803

804

805

806

807

808

809

810

811

812

813

Etherington, K. (2004). Becoming a reflexive researcher. London: Jessica Kingsley publisher.

Fifer, A., Henschen, K., Gould, D., Ravizza, K. (2008). What works when working with athletes. The Sport Psychologist, 22, 356-377.

Gillham, B. (2008). Observation techniques: Structured to unstructured. London: Continuum International Publishing House.

Hall, E. T., Gray, S., \& Sproule, J. (2016). The microstructure of coaching practice: Behaviours and activities of an elite rugby union head coach during preparation and competition. Journal of Sports Sciences, 34, 896-905.

Hays, K., \& Brown, C. (2004). You're on! Consulting for peak performance. Washington, DC: American Psychological Association.

Hemmings, B., \& Holder, T. (2009). Applied sport psychology: A case-based approach. Cornwall, UK: Wiley-Blackwell.

Holder, T., \& Winter, S. (2016). Experienced practitioners use of observation in applied sport psychology. Sport, Exercise, and Performance Psychology. doi:10.1037/spy0000072

Hopkins, P. E. (2007). Thinking critically and creatively about focus groups. Area, 39.4, 528535.

Katz, J., \& Hemmings, B. (2009). Counselling skills handbook for the sport psychologist. Leicester: The British Psychological Society.

Kutz, M. R., Bamford-Wade, A. (2013). Understanding contextual intelligence: A critical competency for today's leaders. $E: C O, 15,55-80$.

Liamputtong, P. (2011). Focus group methodology: Principles and practice. London: Sage.

Litosseliti, L. (2003). Using focus groups in research. London: Continuum. 
Lubker, J. R., Visek, A. J., Geer, J. R., \& Watson, J. C. (2008). Characteristics of an effective sport psychology consultant: Perspectives from athletes and consultants. Journal of Sport Behaviour, 31, 147-165.

Madan, R. M., Conn, D., Dubo, E., Voore, P., \& Wiesenfeld, M. D. (2012). The enablers and barriers to the use of direct observation of trainee clinical skills by supervising faculty in a psychiatry residency program. The Canadian Journal of Psychiatry, 57, 269-272.

McMorris, T. (2015). The practice session: Creating a learning environment. In C. Nash (Ed.), Practical sports coaching (pp. 85-109). Oxon: Routledge.

Milne, D., \& Reiser, R. P. (2011). Observing competence in CBT supervision: A systematic review of the available instruments. The Cognitive Behaviour Therapist, 4, 89-100. doi: $10.1017 / \mathrm{S} 1754470 \mathrm{X} 11000067$

Murphy, S. M., \& Murphy, A. I. (2010). Attending and listening. In S. J. Hanrahan, \& M. B. Anderson (Eds.), Routledge handbook of sport psychology: A comprehensive guide for students and practitioners (pp. 12-20). Oxon: Routledge.

Orlick, T. (1989). Reflections on sportpsych consulting with individual and team sport athletes at summer and winter Olympic Games. The Sport Psychologist, 3, 358-365.

Pain, M. A., \& Harwood, C. G. (2004). Knowledge and perceptions of sport psychology within English soccer. Journal of Sport Sciences, 22, 813-826. doi: $10.1080 / 0264041041000171670$

Palmer, M., Larkin, M., De Visser, R., \& Fadden, G. (2010). Developing an interpretative phenomenological approach to focus group data. Qualitative Research in Psychology, 7, 99-121. doi:10.180/14780880802513194

Partington, J., \& Orlick, T. (1987).The sport psychology consultant: Olympic coaches' views. The Sport Psychologist, 1, 95-102.

Patton, M. Q. (2002). Qualitative research and evaluation methods ( $3^{\text {rd }}$ Ed.). London: Sage. 
Poczwardowski, A., Sherman, C. P., \& Ravizza, K. (2004). Professional philosophy in sport psychology service delivery: Building on theory and practice. The Sport Psychologist, $18,445-463$.

Pringle, J., Drummond, J., McLafferty, E., \& Hendry, C. (2011). Interpretative phenomenological analysis: A discussion and critique. Nurse Researcher, 18, 20-24.

Sharp, L., Hodge, K., \& Danish, S. (2015). Ultimately it comes down to the relationship: Experienced consultants' views of effective sport psychology consulting. The Sport Psychologist, 29, 358-370. doi: 10.1123/tsp.2014-0130

Smith, A. C., \& Sparkes, B. S. (2014). Qualitative research methods in sport, exercise and health: From process to product. Oxon: Routledge.

Smith, J. A. (1996). Beyond the divide between cognition and discourse: Using interpretive phenomenological analysis in health psychology. Psychology and Health, 11, 261271. doi:10.1080/08870449608400256

Smith, J. A., \& Eatough, V. (2012). Interpretative phenomenological analysis. In G. M. Breakwell, J. A. Smith, \& D. B. Wright (Eds.), Research methods in psychology. New Delhi: Sage.

Smith, N., Tessier, D., Tzioumakis, Y., Quested, E., Appleton, P., Sarrazin, P., Papaioannou, A., \& Duda, J. (2015). Development and validation of the multidimensional motivational climate observation system. Journal of Sport and Exercise Psychology, 37, 4-22. doi:10.1123/jsep.2014-0059

Taylor, J., \& Schneider, B. A. (1992). The sport-clinical intake protocol: A comprehensive interviewing instrument for applied sport psychology. Professional Psychology Research and Practice, 23, 318-325.

Tkachuk, G., Leslie-Toogood, L., \& Martin, G.L. (2003). Behavioural assessment in sport psychology. The Sport Psychologist, 17, 104-117. 
TRAINEE EXPERIENCES OF OBSERVATION

864

865

866

867

868

869

870

871

872

873

874

875

876

877

878

879

880

881

882

883

884

885

Tomkins, L., \& Eatough, V. (2010). Reflecting on the use of IPA with focus groups: Pitfalls and potentials. Qualitative Research in Psychology, 7, 244-262. doi:10.1080/14780880903121491

Van Raalte, J. L., Brewer, B. W., Rivera, P. M., \& Petitpas, A. J. (1994). The relationship between observable self-talk and competitive junior tennis players' match performances. Journal of Sport and Exercise Psychology, 16, 400-415.

Vicary, S., Young, A., \& Hicks, S. (2016). A reflective journal as learning process and contribution to quality and validity in interpretative phenomenological analysis. Qualitative Social Work, 0, 1-16.

Watson II, J. C., \& Shannon, V. (2010). Individual and group observation. Purposes and processes. In S. J. Hanrahan, \& M. B. Anderson (Eds.), Routledge handbook of sport psychology: A comprehensive guide for students and practitioners. Oxon: Routledge.

Willis, K., Green, J., Daly, J., Williamson, L., \& Bandyopadhyay, M. (2009). Perils and possibilities: Achieving best evidence from focus groups in public health research. Australian and New Zealand Journal of Public Health, 33, 131-136.

Winter, S., \& Collins, D. (2015a). Where is the evidence in our sport psychology practice? A UK perspective on the underpinnings of action. Professional Psychology: Research and Practice, 46, 175-182. doi:10/1037/pro0000014

Winter, S., \& Collins, D. (2015b). Why do we do, what we do? Journal of Applied Sport Psychology, 27, 35-51. doi:10.1080/10413200.2014.941511.

Winter, S., \& Collins, D. (2016). Applied sport psychology: A profession? The Sport Psychologist, 30, 89-96. doi:10.1123/tsp.2014-0132 